\title{
On the applications of Laplace and Sumudu transforms.
}

\begin{abstract}
In this paper, we study the properties of Sumudu transform and relationship between Laplace and Sumudu transforms. Further, we also provide an example of the double Sumudu transform in order to solve the wave equation in one dimension which is having singularity at initial conditions.
\end{abstract}

Keyword: Convolution; Double convolution; Double Laplace transform; Double Sumudu transform. 